\title{
Analysis of a Model Arising from Invasion by Precursor and Differentiated Cells
}

\author{
Xiaojie Hou \\ Department of Mathematics, University of North Carolina Wilmington, Wilmington, NC 28403, USA
}

Correspondence should be addressed to Xiaojie Hou; houx@uncw.edu

Received 9 April 2013; Accepted 7 August 2013

Academic Editor: Zhi-Qiang Wang

Copyright (C) 2013 Xiaojie Hou. This is an open access article distributed under the Creative Commons Attribution License, which permits unrestricted use, distribution, and reproduction in any medium, provided the original work is properly cited.

We study the wave solutions for a degenerated reaction diffusion system arising from the invasion of cells. We show that there exists a family of waves for the wave speed larger than or equal a certain number and below which there are no monotonic wave solutions. We also investigate the monotonicity, uniqueness, and asymptotics of the waves.

\section{Introduction}

In [1], the following coupled partial differential equation system was proposed to study the invasion by precursor and differentiated cells:

$$
\begin{gathered}
u_{t}=D u_{x x}+\alpha u\left(1-\frac{u+v v}{k_{1}}\right)-\beta u\left(1-\frac{v}{k_{2}}\right), \\
v_{t}=\beta u\left(1-\frac{v}{k_{2}}\right),
\end{gathered}
$$

where $u(x, t)$ denotes the population densities of the precursor cells. The constant $D>0$ is the diffusion rate of the cell $u$, which has proliferation rate $\alpha>0$, and $k_{1}>$ 0 is the carrying capacity of $u$. The parameter $v$ measures the relative contribution that the differentiated cell with population density $v(x, t)$ makes to the carrying capacity $k_{1}$. The cell population density $v$ is limited by its carrying capacity $k_{2}$ and has a maximum differentiation rate $\beta>0$. The model assumes that the differentiated cells do not have mobility.

By letting (see [1])

$$
\widehat{u}=\frac{u}{k_{1}}, \quad \widehat{v}=\frac{v}{k_{1}}, \quad \widehat{t}=\alpha t, \quad \widehat{x}=\sqrt{\frac{\alpha}{D}} x
$$

and dropping the hat notation for convenience, system (1) is changed into

$$
\begin{gathered}
u_{t}=u_{x x}+u(1-u-v v)-\lambda u(1-K v), \\
v_{t}=\lambda u(1-K v),
\end{gathered}
$$

where $\lambda=\beta / \alpha$ and $K=k_{1} / k_{2}$.
System (1) or (3) belongs to reaction diffusion systems of degenerate type, and such systems have attracted much attention in research fields such as epidemics and wound healing [2-4] as well as combustion and calcium wave problems [5-8]. However, system (3) differs from the above systems in the appearance of degenerate reaction terms. In fact, $u=0$ coupling with any $v=$ constant consists of a constant solution of (3). This resembles the combustion wave equation considered in [9]; however, our method in proving the existence of the fronts of (3) differs from theirs.

If the parameters satisfy

$$
0 \leq v<K,
$$

then system (3) admits an additional equilibrium: $B$ : (1 $v / K, 1 / K)$ representing the state that the spatial domain is successfully invaded. We also separate the equilibrium $A$ : $(0,0)$ from the rest of the line of equilibria, $u=0$. The unstable equilibrium $(0,0)$ represents the state before the invasion.

We are interested in the existence of the wave solutions connecting $A$ with $B$ as time and space evolve from $-\infty$ to $+\infty$. Setting $\xi=x+c t, x \in \mathbb{R}, t \in \mathbb{R}^{+}$, a traveling wave solution to (3) solves

$$
\begin{gathered}
u_{\xi \xi}-c u_{\xi}+u(1-u-v v)-\lambda u(1-K v)=0, \\
-c v_{\xi}+\lambda u(1-K v)=0
\end{gathered}
$$


with boundary conditions:

$$
(u, v)(-\infty)=(0,0), \quad(u, v)(+\infty)=\left(1-\frac{v}{K}, \frac{1}{K}\right) .
$$

For the notational convenience, we further set

$$
\bar{u}=\frac{u}{1-v / K},
$$

and drop the bar on $u$ to have

$$
\begin{gathered}
u_{\xi \xi}-c u_{\xi}+u\left[1-\lambda-\left(1-\frac{v}{K}\right) u+(\lambda K-v) v\right]=0, \\
-c v_{\xi}+\lambda\left(1-\frac{v}{K}\right) u(1-K v)=0, \\
(u, v)(-\infty)=(0,0), \quad(u, v)(+\infty)=\left(1, \frac{1}{K}\right) .
\end{gathered}
$$

Numerical investigations [1] strongly suggest that system (10) and (9) admit traveling wave solutions for $v=0$ and $v=1$. When the differentiated cell density does not affect the proliferation of the precursor cells, we have $v=0$; when the total cell population contributes to the proliferation carrying capacity, we have $v=1$. Numerically, however, when $v=$ 1 , (8) may have nonmonotone traveling wave solutions and requires a different treatment. Hence, in this paper we only study the wave solutions for $v=0$. The system (8) in this case can be further reduced to

$$
\begin{gathered}
u_{\xi \xi}-c u_{\xi}+u(1-\lambda-u+\lambda K v)=0, \\
-c v_{\xi}+\lambda u(1-K v)=0 .
\end{gathered}
$$

The computations in [1] show that the wave may exist for $c \geq 2 \sqrt{1-\lambda}$, but a rigorous existence proof is still lacking. We will confirm this observation by a mathematical analysis of the model. The system is of cooperative type, and we can use the monotone iteration scheme developed in [10] for the existence proof. Such method reduces the existence of the wave solutions to that of the ordered upper and lower solution pairs for (10) and (9). The upper and lower solutions in this paper come directly from two KPP type equations, which are constructed so that they have the same decay rate at $-\infty$. Such information is also relevant to the monotonicity and uniqueness of the wave solutions. Indeed, since we have a good understanding of the decay properties of the solutions at infinities, we then can study the properties of the solutions on finite domain, in which the powerful sliding domain method (see [11]) can be used to have the desired results. We remark that the methods we used in the proofs of the monotonicity and the uniqueness have subtle difference from the ones used in [12].

For a comprehensive study and interesting applications of the traveling wave solutions arising in various degenerate or nondegenerate parabolic equations and systems, please see [13].

\section{The Main Result}

In this section, we will use monotone iteration method to set up the upper and lower solutions for system (10) and (9).

Definition 1. A $C^{2}(\mathbb{R}) \times C^{1}(\mathbb{R})$ function $(\bar{u}(\xi), \bar{v}(\xi))^{T}, \xi \in \mathbb{R}$ is an upper solution of (10) and (9) if it satisfies

$$
\begin{gathered}
u_{\xi \xi}-c u_{\xi}+u(1-\lambda-u+\lambda K v) \leq 0, \\
-c v_{\xi}+\lambda u(1-K v) \leq 0
\end{gathered}
$$

and the boundary conditions

$$
\left(\begin{array}{l}
u \\
v
\end{array}\right)(-\infty) \geq\left(\begin{array}{l}
0 \\
0
\end{array}\right), \quad\left(\begin{array}{c}
u \\
v
\end{array}\right)(+\infty) \geq\left(\begin{array}{c}
1 \\
\frac{1}{K}
\end{array}\right) .
$$

We can similarly define the lower solution $(\underline{u}, \underline{v})(\xi), \xi \in \mathbb{R}$ by reversing the inequalities (11) and (12).

The following known result [14] is needed in the construction of the upper and lower solutions.

Consider the following form of the KPP equation:

$$
\begin{gathered}
\omega^{\prime \prime}-c \omega^{\prime}+f(\omega)=0, \\
\omega(-\infty)=0, \quad \omega(+\infty)=b,
\end{gathered}
$$

where $f \in C^{2}([0, b])$ and $f>0$ on the open interval $(0, b)$ with $f(0)=f(b)=0, f^{\prime}(0)=\bar{a}>0$, and $f^{\prime}(b)=-b_{1}<0$.

Lemma 2. Corresponding to every $c \geq 2 \sqrt{\bar{a}}$, system (13) has a unique (up to a translation of the origin) monotonically increasing traveling wave solution $\omega(\xi)$ for $\xi \in \mathbb{R}$. The traveling wave solution $\omega$ has the following asymptotic behaviors.

For the wave solution with noncritical speed $c>2 \sqrt{\bar{a}}$, one has

$$
\begin{aligned}
& \omega(\xi)=\bar{a}_{\omega} e^{\left(\left(c-\sqrt{c^{2}-4 \bar{a}}\right) / 2\right) \xi}+o\left(e^{\left(\left(c-\sqrt{c^{2}-4 \bar{a}}\right) / 2\right) \xi}\right) \quad \text { as } \xi \longrightarrow-\infty, \\
& \omega(\xi)=b-\bar{b}_{\omega} e^{\left(\left(c-\sqrt{c^{2}+4 b_{1}}\right) / 2\right) \xi} \\
& +o\left(e^{\left(\left(c-\sqrt{c^{2}+4 b_{1}}\right) / 2\right) \xi}\right) \quad \text { as } \xi \longrightarrow+\infty \text {, }
\end{aligned}
$$

where $\bar{a}_{\omega}$ and $\bar{b}_{\omega}$ are positive constants.

For the wave with critical speed $c=2 \sqrt{\bar{a}}$, one has

$$
\begin{gathered}
\omega(\xi)=\bar{d}_{c} \xi e^{\sqrt{\bar{a} \xi}}+o\left(\xi e^{\sqrt{\bar{a} \xi}}\right) \quad \text { as } \xi \longrightarrow-\infty, \\
\omega(\xi)=b-\bar{b}_{c} e^{\left(\sqrt{\bar{a}}-\sqrt{\bar{a}+b_{1}}\right) \xi}+o\left(e^{\left(\sqrt{\bar{a}}-\sqrt{\bar{a}+b_{1}}\right) \xi}\right) \quad \text { as } \xi \longrightarrow+\infty,
\end{gathered}
$$

where the constant $\bar{d}_{c}$ is negative and $\bar{b}_{c}$ is positive.

We next consider the following version of the KPP system:

$$
\begin{gathered}
\omega^{\prime \prime}-c \omega^{\prime}+(1-\lambda) \omega(1-\omega)=0, \\
\omega(-\infty)=0, \quad \omega(+\infty)=1 .
\end{gathered}
$$


According to Lemma 2, for every $c \geq 2 \sqrt{1-\lambda}$ system, (16) has a unique (up to a translation of the origin) monotone solution $\widetilde{u}(\xi), \xi \in \mathbb{R}$. Now, fix this $\widetilde{u}(\xi)$ and consider the equation

$$
-c v_{\xi}+\lambda \tilde{u}(1-K v)=0 .
$$

For each fixed $c \geq 2 \sqrt{1-\lambda}$ and the corresponding $\widetilde{u}(\xi)$, (17) has a solution

$$
\bar{v}(\xi)=\frac{1}{K}\left(1-e^{-(\lambda / c) K \int_{-\infty}^{\xi} \tilde{u}(s) d s}\right) .
$$

We next compare $\widetilde{u}(\xi)$ with $\bar{v}(\xi)$ for $\xi \in \mathbb{R}$.

Lemma 3. There exists $a \zeta_{1} \geq 0$ such that if

$$
0<\lambda \leq \frac{2}{2+K(1+\sqrt{2})},
$$

one has

$$
\frac{1}{K} \widetilde{u}\left(\xi+\zeta_{1}\right) \geq \bar{v}(\xi), \quad \xi \in \mathbb{R}
$$

Proof. According to Lemma 2, the wave solution $\widetilde{u}(\xi)$ to (16) has the following asymptotic behaviors.

For $c>2 \sqrt{1-\lambda}$,

$$
\begin{aligned}
\tilde{u}(\xi)= & a_{\omega} e^{\left(\left(c-\sqrt{c^{2}-4(1-\lambda)}\right) / 2\right) \xi} \\
& +o\left(e^{\left(\left(c-\sqrt{c^{2}-4(1-\lambda)}\right) / 2\right) \xi}\right) \text { as } \xi \longrightarrow-\infty, \\
\tilde{u}(\xi)= & \left.b-b_{\omega} e^{\left(\left(c-\sqrt{c^{2}+4(1-\lambda)}\right) / 2\right.}\right) \xi \\
& +o\left(e^{\left(\left(c-\sqrt{c^{2}+4(1-\lambda)}\right) / 2\right) \xi}\right) \text { as } \xi \longrightarrow+\infty,
\end{aligned}
$$

and $a_{\omega}, b_{\omega}$ are positive constants.

For $c=2 \sqrt{1-\lambda}$, we have

$$
\begin{gathered}
\tilde{u}(\xi)=d_{c} \xi e^{\sqrt{1-\lambda} \xi}+o\left(\xi e^{\sqrt{1-\lambda} \xi}\right) \quad \text { as } \xi \longrightarrow-\infty, \\
\tilde{u}(\xi)=b-b_{c} e^{(1-\sqrt{2}) \sqrt{1-\lambda} \xi}+o\left(e^{(1-\sqrt{2}) \sqrt{1-\lambda} \xi}\right) \quad \text { as } \xi \longrightarrow+\infty,
\end{gathered}
$$

where the constant $d_{c}$ is negative and $b_{c}$ is positive.

We now study the asymptotics of the function $\bar{v}(\xi)$. Formulas (21) and (23) imply that

$$
\int_{-\infty}^{\xi} \tilde{u}(s) d s \longrightarrow 0 \quad \text { as } \xi \longrightarrow-\infty .
$$

We can then expand

$$
\begin{aligned}
e^{-(\lambda / c) K \int_{-\infty}^{\xi} \widetilde{u}(s) d s}= & 1-\frac{\lambda}{c} K \int_{-\infty}^{\xi} \tilde{u}(s) d s \\
& +o\left(\left(\int_{-\infty}^{\xi} \tilde{u}(s) d s\right)^{2}\right) .
\end{aligned}
$$

A further expanding of (26) for $\xi \rightarrow-\infty$ and for $c>$ $2 \sqrt{1-\lambda}$ yields

$$
\begin{aligned}
1- & e^{-(\lambda / c) K \int_{-\infty}^{\xi} \tilde{u}(s) d s} \\
= & \frac{2 \lambda a_{\omega}}{\left(c-\sqrt{c^{2}-4(1-\lambda)}\right) c} e^{\left(\left(c-\sqrt{c^{2}-4(1-\lambda)}\right) / 2\right) \xi} \\
& +o\left(e^{\left(\left(c-\sqrt{c^{2}-4(1-\lambda)}\right) / 2\right) \xi}\right)
\end{aligned}
$$

and for $c=2 \sqrt{1-\lambda}$,

$$
\begin{aligned}
1- & e^{-(\lambda / c) K \int_{-\infty}^{\xi} \widetilde{u}(s) d s} \\
& =\frac{\lambda}{2 \sqrt{1-\lambda}}\left(d_{c} \xi-\frac{d_{c}}{1-\lambda}\right) e^{\sqrt{1-\lambda} \xi}+o\left(\xi e^{\sqrt{1-\lambda} \xi}\right) .
\end{aligned}
$$

As for $\xi>0$ sufficiently large, we have

$$
\lim _{\xi \rightarrow+\infty} \frac{\int_{-\infty}^{\xi} \tilde{u}(s) d s}{\xi}=\lim _{\xi \rightarrow+\infty} \tilde{u}(\xi)=1 ;
$$

therefore,

$$
\bar{v}(\xi)=K\left(1-e^{-(\lambda / c) K \xi}\right)+o\left(e^{-(\lambda / c) K \xi}\right) \quad \text { as } \xi \longrightarrow+\infty .
$$

We next show

$$
-\frac{\lambda}{c} K \geq \frac{c-\sqrt{c^{2}+4(1-\lambda)}}{2}
$$

or equivalently

$$
\frac{2 c(1-\lambda)}{c+\sqrt{c^{2}+4(1-\lambda)}} \geq \lambda K .
$$

Setting $g(c)=2(1-\lambda) / 1+\sqrt{1+4(1-\lambda) / c^{2}}$, then it is easy to see that $g(c)$ increases as $c$ does. Hence,

$$
g(c) \geq g(2 \sqrt{1-\lambda})=\frac{2(1-\lambda)}{1+\sqrt{2}} .
$$

We therefore require $0<\lambda \leq 2 /(2+K(1+\sqrt{2}))$ to have (31).

We now shift $\widetilde{u}(\xi)$. Since (16) is shifting invariant, $\widetilde{u}(\xi+\zeta)$, $\xi \in \mathbb{R}$ is also a solution for any $\zeta \in \mathbb{R}$. It then follows from (21) for $c>\sqrt{1-\lambda}$,

$$
\begin{aligned}
\tilde{u}(\xi+\zeta)= & a_{\omega} e^{\left(\left(c-\sqrt{c^{2}-4(1-\lambda)}\right) / 2\right) \zeta} e^{\left(\left(c-\sqrt{c^{2}-4(1-\lambda)}\right) / 2\right) \xi} \\
& +o\left(e^{\left(\left(c-\sqrt{c^{2}-4(1-\lambda)}\right) / 2\right) \xi}\right) \quad \text { as } \xi \longrightarrow-\infty
\end{aligned}
$$

and for $c=\sqrt{1-\lambda}$,

$$
\begin{aligned}
\tilde{u}(\xi+\zeta)= & d_{c}(\xi+\zeta) e^{\sqrt{1-\lambda} \zeta} e^{\sqrt{1-\lambda} \xi} \\
& +o\left(\xi e^{\sqrt{1-\lambda} \xi}\right) \quad \text { as } \xi \longrightarrow-\infty
\end{aligned}
$$


If we choose $\zeta>0$ sufficiently large, the positiveness of $\left(\left(c-\sqrt{c^{2}-4(1-\lambda)}\right) / 2\right) \zeta$ and $\sqrt{1-\lambda} \zeta$ implies that if $c=$ $2 \sqrt{1-\lambda}$,

$$
d_{c}(\xi+\zeta) e^{\sqrt{1-\lambda} \zeta}>\frac{\lambda}{2 \sqrt{1-\lambda}}\left(d_{c} \xi-\frac{d_{c}}{1-\lambda}\right)
$$

and if $c>2 \sqrt{1-\lambda}$,

$$
a_{\omega} e^{\left(\left(c-\sqrt{\left.c^{2}-4(1-\lambda)\right) / 2}\right) \zeta\right.}>\frac{2 \lambda a_{\omega}}{\left(c-\sqrt{c^{2}-4(1-\lambda)}\right) c} .
$$

It then follows from (31), (34), and (35) that there exists a $\bar{N}>0$ sufficiently large,

$$
\frac{1}{K} \widetilde{u}(\xi+\zeta) \geq \bar{v}(\xi) \quad \text { for } \xi \in(-\infty,-N] \cup[N,+\infty),
$$

and for $\xi \in[-N, N]$, since $\widetilde{u}$ and $\bar{v}$ are both monotonically increasing on $\mathbb{R}$ we can further shift $\tilde{u}(\xi+\zeta)$ to the left at most $2 N$ units to have $(1 / K) \widetilde{u}(\xi+\zeta) \geq \bar{v}(\xi), \xi \in \mathbb{R}$. Hence there exists a finite $\zeta_{1} \geq 0$ such that the conclusion of the Lemma holds.

Now, we write $\bar{u}(\xi)=\widetilde{u}\left(\xi+\zeta_{0}\right), \xi \in \mathbb{R}$ and let $\bar{v}(\xi)$ be defined in (18). We remark here that the computation of $\bar{v}(\xi)$ still uses $\widetilde{u}(\xi)$.

Lemma 4. Assume the conditions in Lemma 3, then $(\bar{u}, \bar{v})(\xi)$, $\xi \in \mathbb{R}$ defines an upper solution for (10) and (9).

Proof. We can easily verify that $(\bar{u}, \bar{v})(\xi)$ satisfies the boundary conditions (12).

For the $u$ component, we have

$$
\begin{aligned}
\bar{u}^{\prime \prime} & -c \bar{u}^{\prime}+\bar{u}(1-\lambda-\bar{u}+\lambda K \bar{v}) \\
& =\bar{u}[1-\lambda-\bar{u}+\lambda K \bar{v}-(1-\lambda)(1-\bar{u})] \\
& =-\lambda K \bar{u}\left(\frac{1}{K} \bar{u}-\bar{v}\right) \leq 0 .
\end{aligned}
$$

The last inequality follows from the previous lemma.

As for the $v$ component, for each $\widetilde{u}$, we have

$$
-c \bar{v}_{\xi}+\lambda \tilde{u}(1-K \bar{v})=0 .
$$

We next set up the lower solution for (10) and (9).

For a fixed $l>0$, we consider another version of the KPP system:

$$
\begin{gathered}
w^{\prime \prime}-c w^{\prime}+(1-\lambda) w\left(1-\frac{1-\lambda+l}{1-\lambda} w\right)=0, \\
w(-\infty)=0, \quad w(+\infty)=\frac{1-\lambda}{1-\lambda+l}<1 .
\end{gathered}
$$

Then, for any $c \geq 2 \sqrt{1-\lambda}$, (41) has correspondingly a unique wave solution $\breve{u}(\xi), \xi \in \mathbb{R}$.
We define

$$
\underline{v}(\xi)=\frac{1}{K}\left(1-e^{-(\lambda / c) K \int_{-\infty}^{\xi} \breve{u}(s) d s}\right) .
$$
$\xi \in \mathbb{R}$

The next lemma gives the relation between $\breve{u}(\xi)$ and $\underline{v}(\xi)$,

Lemma 5. There exists a $\zeta_{1} \geq 0$ such that

$$
\frac{1}{K} \breve{u}\left(\xi-\zeta_{1}\right) \leq \underline{v}(\xi), \quad \xi \in \mathbb{R} .
$$

Proof. The proof is similar to that of Lemma 3. Noting as $\xi \rightarrow$ $+\infty, \breve{u}(\xi) \rightarrow(1 / K)((1-\lambda) /(1-\lambda+l))<1 / K$. Hence, we do not need condition (19) here.

We denote $\underline{u}(\xi)=\breve{u}\left(\xi-\zeta_{1}\right), \xi \in \mathbb{R}$. Then, we have the following.

Lemma 6. Such defined $(\underline{u}, \underline{v})(\xi), \xi \in \mathbb{R}$ consists of a lower solution for (10) and (9).

Proof. One the boundary, we have

$$
\begin{gathered}
(\underline{u}, \underline{v})(-\infty)=(0,0), \\
(\underline{u}, \underline{v})(+\infty)=\left(\frac{1-\lambda}{1-\lambda+l}, \frac{1}{K}\right) \leq\left(1, \frac{1}{K}\right)
\end{gathered}
$$

and for the $u$ component,

$$
\begin{aligned}
\underline{u}^{\prime \prime}- & \underline{c} \underline{u}^{\prime}+\underline{u}(1-\lambda-\underline{u}+\lambda K \underline{v}) \\
= & \underline{u}^{\prime \prime}-c \underline{u}^{\prime}+(1-\lambda) \underline{u}\left(1-\frac{1-\lambda+l}{1-\lambda} \underline{u}\right) \\
& -(1-\lambda) \underline{u}\left(1-\frac{1-\lambda+l}{1-\lambda} \underline{u}\right) \\
& +\underline{u}(1-\lambda-\underline{u}+\lambda K \underline{v}) \\
= & \underline{u}[1-\lambda-\underline{u}+\lambda K \underline{v}-(1-\lambda)+(1-\lambda+l) \underline{u}] \geq 0
\end{aligned}
$$

due to Lemma 5 .

Noting that $\underline{v}(\xi)$ solves the equation

$$
-c \underline{v_{\xi}}+\lambda \breve{u}(1-K \underline{v})=0,
$$

it satisfies the inequality trivially.

Lemma 7. The upper and lower solutions are ordered

$$
(\bar{u}, \bar{v})(\xi) \geq(\underline{u}, \underline{v})(\xi), \quad \xi \in \mathbb{R} .
$$

Proof. For each fixed $c \geq 2 \sqrt{1-\lambda}$, if $\breve{u}(\xi)$ solves the system (41), then the function $\widetilde{u}(\xi)=((1-\lambda+l) /(1-\lambda)) \breve{u}(\xi)$ solves (16). Hence, it follows that $\widetilde{u}(\xi)>\breve{u}(\xi)$, and then $\bar{u}(\xi)>\underline{u}(\xi)$ for all $\xi \in \mathbb{R}$.

By definition of $\bar{v}(\xi)$ and $\underline{v}(\xi)$, we have

$$
\begin{aligned}
& \bar{v}(\xi)=\frac{1}{K}\left(1-e^{-(\lambda / c) K \int_{-\infty}^{\xi} \tilde{u}(s) d s}\right) \\
& =\frac{1}{K}\left(1-e^{-(\lambda / c) K((1-\lambda+l) /(1-\lambda)) \int_{-\infty}^{\xi} \breve{u}(s) d s}\right) \\
& >\frac{1}{K}\left(1-e^{-(\lambda / c) K \int_{-\infty}^{\xi} \breve{u}(s) d s}\right)=\underline{v}(\xi) .
\end{aligned}
$$

Hence, the conclusion of the lemma holds. 
Theorem 8. Let the parameters satisfy (19), then for each $c \geq$ $2 \sqrt{1-\lambda}$, system (10) and (9) have a unique (up to a translation of the origin) strictly monotonically increasing traveling wave solution, while for $0<c<2 \sqrt{1-\lambda}$, there is no monotonic traveling wave. The traveling wave solution has the following asymptotic behaviors.

For $c=2 \sqrt{1-\lambda}$,

$$
\left(\begin{array}{l}
u \\
v
\end{array}\right)(\xi)=\left(\begin{array}{l}
c_{11} \xi \\
c_{12} \xi
\end{array}\right) e^{\sqrt{1-\lambda} \xi}+o\left(e^{\sqrt{1-\lambda} \xi}\right), \quad \text { as } \xi \longrightarrow-\infty \text {, }
$$

$$
\begin{aligned}
\left(\begin{array}{c}
u \\
v
\end{array}\right)(\xi)= & \left(\begin{array}{c}
1 \\
\frac{1}{K}
\end{array}\right)-\left(\begin{array}{l}
c_{21} e^{-(\lambda K / 2 \sqrt{1-\lambda}) \xi} \\
c_{22} e^{-(\lambda K / 2 \sqrt{1-\lambda}) \xi}
\end{array}\right) \\
& +\left(\begin{array}{c}
o\left(e^{-(\lambda K / 2 \sqrt{1-\lambda}) \xi}\right) \\
o\left(e^{-(\lambda K / 2 \sqrt{1-\lambda}) \xi}\right)
\end{array}\right), \quad \text { as } \xi \longrightarrow+\infty,
\end{aligned}
$$

and for $c>2 \sqrt{1-\lambda}$,

$$
\begin{aligned}
\left(\begin{array}{l}
u \\
v
\end{array}\right)(\xi)= & \left(\begin{array}{l}
d_{11} \xi \\
d_{12} \xi
\end{array}\right) e^{\left(\left(c-\sqrt{c^{2}+4(1-\lambda)}\right) / 2\right) \xi} \\
& +o\left(e^{\left(\left(c-\sqrt{\left.c^{2}+4(1-\lambda)\right)} / 2\right) \xi\right.}\right), \quad \text { as } \xi \longrightarrow-\infty \\
\left(\begin{array}{l}
u \\
v
\end{array}\right)(\xi)= & \left(\begin{array}{c}
1 \\
\frac{1}{K}
\end{array}\right)-\left(\begin{array}{l}
d_{21} e^{-(\lambda K / c) \xi} \\
d_{22} e^{-(\lambda K / c) \xi}
\end{array}\right) \\
& +\left(\begin{array}{c}
o\left(e^{-(\lambda K / c) \xi}\right) \\
o\left(e^{-(\lambda K / c) \xi}\right)
\end{array}\right) \quad \text { as } \xi \longrightarrow+\infty
\end{aligned}
$$

where $c_{11}, c_{12}, c_{21}, c_{22}, d_{21}$, and $d_{22}>0$ and $d_{11}, d_{12}<0$.

Proof. Noting that between the upper and lower solutions, there is no equilibrium other than $(0,0)$ and $(1,1 / K)$ of system (10) and (9). Hence, the monotone iteration scheme developed in [10] is still applicable. Such monotone iteration scheme reduces the existence of the traveling wave solutions to that of the ordered upper and lower solution pairs, and the existence of the traveling waves then follows by Lemmas 6, 4, and 7 , and by [10], so the obtained traveling wave solutions are nondecreasing, while for $c<2 \sqrt{1-\lambda}$, it is easy to verify, by analyzing the equilibrium $(0,0)$, that the nontrivial bounded solutions of (10) are oscillatory.

We next show that the wave solutions are strictly monotonically increasing on $\mathbb{R}$.

For any fixed $c \geq \sqrt{1-\lambda}$, let $\left(u_{c}, v_{c}\right)(\xi)$ be the corresponding traveling wave solution and $\left(w_{1}(\xi), w_{2}(\xi)\right)$ be its derivative. Then, $\left(w_{1}(\xi), w_{2}(\xi)\right) \geq 0$ for $\xi \in \mathbb{R}$, and $\left(w_{1}(\xi), w_{2}(\xi)\right)$ satisfies the following systems:

$$
\begin{gathered}
w_{1, \xi \xi}-c w_{1, \xi}+\left(1-\lambda-2 u_{c}+\lambda K v_{c}\right) w_{1}+\lambda K u_{c} w_{2}=0, \\
-c w_{2, \xi}+\lambda\left(1-K v_{c}\right) w_{1}-\lambda K u_{c} w_{2}=0, \\
\left(w_{1}, w_{2}\right)( \pm \infty)=0 .
\end{gathered}
$$

It then follows that

$$
\begin{gathered}
w_{1, \xi \xi}-c w_{1, \xi}+\left(1-\lambda-2 u_{c}+\lambda K v_{c}\right) w_{1} \leq 0, \\
-c w_{2, \xi}+\lambda\left(1-K v_{c}\right) w_{1}-\lambda K u_{c} w_{2}=0, \\
\left(w_{1}, w_{2}\right)( \pm \infty)=0 .
\end{gathered}
$$

Applying the maximum principle to the first inequality of (54), we immediately conclude that $w_{1}(\xi)>0$ for $\xi \in \mathbb{R}$. Thu, $u_{c}(\xi)$ is strictly monotonically increasing.

The strict monotonicity of $v_{c}(\xi)$ comes from (10). Since $u_{c}(\xi)>0$ for all $\xi \in \mathbb{R}$, and for such $u_{c}(\xi)$, we have

$$
v_{c}(\xi)=\frac{1}{K}\left(1-e^{-(\lambda / c) K \int_{-\infty}^{\xi} u_{c}(s) d s}\right),
$$

then it follows that $w_{2}(\xi)=v_{c}^{\prime}(\xi)>0, \xi \in \mathbb{R}$. This shows that the wave solution $\left(u_{c}, v_{c}\right)$ is strictly monotonically increasing.

We then derive the asymptotics of the wave solutions at $\pm \infty$. Noting that the upper and lower solutions have the same exponential decay rate at $-\infty,(49)$ and (51) come directly from comparison.

We next study the asymptotics of the function $\left(w_{1}, w_{2}\right)(\xi)$ at $+\infty$, recalling that $\left(w_{1}, w_{2}\right)(\xi)=\left(u_{c}, v_{c}\right)^{\prime}(\xi)$ and that it satisfies the system (53). Since this system is hyperbolic at $+\infty,\left(w_{1}, w_{2}\right)$ approaches $(0,0)$ exponentially. We will derive the exact exponential rate.

The limit equation at $+\infty$ of system (53) is

$$
\begin{gathered}
w_{1, \xi \xi}^{+}-c w_{1, \xi}^{+}-w_{1}^{+}+\lambda K w_{2}^{+}=0, \\
-c w_{2, \xi}^{+}-\lambda K u_{c} w_{2}^{+}=0 .
\end{gathered}
$$

Since the second equation is decoupled from the system, we immediately have

$$
w_{2}^{+}(\xi)=\underline{A} e^{-(\lambda K / c) \xi} .
$$

Plugging the above into the first equation yields a bounded solution (up to the first order approximation) of the form

$$
w_{1}^{+}(\xi)=\bar{A}_{1} e^{-(\lambda K / c) \xi}+\bar{A}_{2} e^{((c-\sqrt{c+4}) / 2) \xi} .
$$

By roughness of exponential dichotomy [15], we have

$$
\left(\begin{array}{l}
w_{1}(\xi) \\
w_{2}(\xi)
\end{array}\right)=\left(\begin{array}{c}
\frac{A_{1} e^{-(\lambda K / c) \xi}}{\bar{A}_{2} e^{\mu \xi}}
\end{array}\right)+\left(\begin{array}{c}
o\left(e^{-(\lambda K / c) \xi}\right) \\
o\left(e^{\mu \xi}\right)
\end{array}\right)
$$

where $\mu$ is either $-\lambda K / c$ or $(c-\sqrt{c+4}) / 2$. 
Integrating the above from $\xi_{0}$ to $+\infty$ and comparing the decay rates of $\left(u_{c}, v_{c}\right)(\xi)$ with that of the upper solution $(\bar{u}, \bar{v})(\xi)$, we have $(50)$ and $(52)$.

On the uniqueness of the traveling wave solution for every $c \geq 2 \sqrt{1-\lambda}$, we only prove the conclusion for traveling wave solutions with asymptotic rates given in (51) and (52) since the other case can be proved similarly. Let $U_{1}(\xi)=\left(u_{1}, v_{1}\right)(\xi)$ and $U_{2}(\xi)=\left(u_{2}, v_{2}\right)(\xi)$ be two traveling wave solutions of system (10) and (9) with the same speed $c>2 \sqrt{1-\lambda}$. There exist positive constants $A_{i j}, B_{i j}, i, j=1,2$ and a large number $N>0$ such that for $\xi<-N$,

$$
\begin{aligned}
& U_{1}(\xi)=\left(\begin{array}{l}
A_{11} \\
A_{12}
\end{array}\right) e^{\left(\left(c+\sqrt{c^{2}-4(1-\lambda)}\right) / 2\right) \xi}+o\left(e^{\left(\left(c+\sqrt{c^{2}-4(1-\lambda)}\right) / 2\right) \xi}\right) \\
& U_{2}(\xi)=\left(\begin{array}{l}
A_{21} \\
A_{22}
\end{array}\right) e^{\left(\left(c+\sqrt{\left.c^{2}-4(1-\lambda)\right)} / 2\right) \xi\right.}+o\left(e^{\left(\left(c+\sqrt{c^{2}-4(1-\lambda)}\right) / 2\right) \xi}\right)
\end{aligned}
$$

and for $\xi>N$,

$$
\begin{aligned}
& U_{1}(\xi)=\left(\begin{array}{c}
1-B_{11} e^{-(\lambda / c) K \xi} \\
\frac{1}{K}-B_{12} e^{-(\lambda / c) K \xi}
\end{array}\right)+\left(\begin{array}{c}
o\left(e^{-(\lambda / c) K \xi}\right) \\
\left(e^{-(\lambda / c) K \xi}\right)
\end{array}\right), \\
& U_{2}(\xi)=\left(\begin{array}{c}
1-B_{21} e^{-(\lambda / c) K \xi} \\
\frac{1}{K}-B_{22} e^{-(\lambda / c) K \xi}
\end{array}\right)+\left(\begin{array}{c}
o\left(e^{-(\lambda / c) K \xi}\right) \\
\left(e^{-(\lambda / c) K \xi}\right)
\end{array}\right) .
\end{aligned}
$$

The traveling wave solutions of system (10)-(9) are translation invariant; thus, for any $\theta>0, U_{1}^{\theta}(\xi):=U_{1}(\xi+\theta)$ is also a traveling wave solution of (10)-(9). By (60) and (62), the solution $U_{1}(\xi+\theta)$ has the asymptotics

$$
\begin{aligned}
U_{1}^{\theta}(\xi)= & \left(\begin{array}{l}
A_{11} e^{\left(\left(c+\sqrt{c^{2}-4(1-\lambda)}\right) / 2\right) \theta} \\
A_{12} e^{\left(\left(c+\sqrt{c^{2}-4(1-\lambda)}\right) / 2\right) \theta}
\end{array}\right) e^{\left(\left(c+\sqrt{\left.c^{2}-4(1-\lambda)\right)} / 2\right) \xi\right.} \\
& +o\left(e^{\left(\left(c+\sqrt{c^{2}-4(1-\lambda)}\right) / 2\right) \xi}\right)
\end{aligned}
$$

for $\xi \leq-N$ and

$$
U_{1}^{\theta}(\xi)=\left(\begin{array}{c}
1-B_{11} e^{-(\lambda / c) K \theta} e^{-(\lambda / c) K \xi} \\
\frac{1}{K}-B_{12} e^{-(\lambda / c) K \theta} e^{-(\lambda / c) K \xi}
\end{array}\right)+\left(\begin{array}{c}
o\left(e^{-(\lambda / c) K \xi}\right) \\
\left(e^{-(\lambda / c) K \xi}\right)
\end{array}\right)
$$

for $\xi \geq N$.

Choosing $\theta>0$ large enough such that

$$
\begin{gathered}
A_{11} e^{\left(\left(c+\sqrt{\left.c^{2}-4(1-\lambda)\right)} / 2\right) \theta\right.}>A_{21}, \\
A_{12} e^{\left(\left(c+\sqrt{\left.c^{2}-4(1-\lambda)\right)} / 2\right) \theta\right.}>A_{22}, \\
B_{11} e^{-(\lambda / c) K \theta}<B_{21}, \\
B_{12} e^{-(\lambda / c) K \theta}<B_{22},
\end{gathered}
$$

then one has for $\xi \in(-\infty,-N] \cup[N,+\infty)$,

$$
U_{1}^{\theta}(\xi)>U_{2}(\xi) .
$$

We now consider system $(10)$ on $[-N,+N]$. There are two possibilities.

Case 1. Suppose that we already have $U_{1}^{\theta}(\xi) \geq U_{2}(\xi)$ on $[-N,+N]$, then the function $W(\xi)=\left(w_{1}(\xi), w_{2}(\xi)\right)^{T}:=$ $U_{1}^{\theta}(\xi)-U_{2}(\xi) \geq 0$ and it satisfies for some $\zeta_{i} \in(0,1), i=1,2$,

$$
\left(\begin{array}{c}
w_{1}^{\prime \prime} \\
0
\end{array}\right)-c\left(\begin{array}{l}
w_{1}^{\prime} \\
w_{2}^{\prime}
\end{array}\right)+M\left(\begin{array}{l}
w_{1} \\
w_{2}
\end{array}\right)=0,
$$

$$
W(-N)>0, \quad W(+N)>0 . \quad \xi \in(-N, N),
$$

where the matrix $M$ is given by

$$
\begin{aligned}
& M\left(w_{1}, w_{2}\right) \\
& \quad=\left(\begin{array}{cc}
1-\lambda-2\left(u_{2}+\zeta_{1} w_{1}\right)+\lambda K\left(v_{2}+\zeta_{2} w_{2}\right), & \lambda K\left(u_{2}+\zeta_{1} w_{1}\right) \\
\lambda\left(1-K\left(v_{2}+\zeta_{2} w_{2}\right)\right), & -\lambda K\left(u_{2}+\zeta_{2} w_{1}\right)
\end{array}\right) .
\end{aligned}
$$

Since $w_{1}(\xi) \geq 0, \xi \in[-N, N]$ and $\lambda K\left(u_{2}+\zeta_{1} w_{1}\right) \geq 0$, then we have on $\xi \in[-N, N]$,

$$
\begin{aligned}
w_{1}^{\prime \prime}-c w_{1}^{\prime}+ & {\left[1-\lambda-2\left(u_{2}+\zeta_{1} w_{1}\right)+\lambda K\left(v_{2}+\zeta_{2} w_{2}\right)\right] w_{1} } \\
+ & \lambda K\left(u_{2}+\zeta_{2} w_{1}\right) w_{2}=0 \\
& w_{1}(-N)>0, \quad w_{1}(N)>0 .
\end{aligned}
$$

The maximum principle then implies that $w_{1}(\xi)>0$ on $[-N, N]$. We then move to the second equation of $(68)$. We have

$$
\begin{aligned}
& -c w_{2}^{\prime}-\lambda K\left(u_{2}+\zeta_{2} w_{1}\right) w_{2} \\
& =-\lambda\left(1-K\left(v_{2}+\zeta_{2} w_{2}\right)\right) w_{1}<0, \quad \xi \in[-N, N], \\
& w_{2}(-N)>0, \quad w_{2}(N)>0 .
\end{aligned}
$$

The strict inequality comes from the fact that $v_{2}(\xi) \leq$ $v_{2}(\xi)+\zeta_{2} w_{2}(\xi) \leq v_{1}^{\theta}<1 / K$ for $\xi \in[-N, N]$. It then follows that $w_{2}(\xi)>0$ for $\xi \in[-N, N]$. For if there is a $\bar{\xi} \in(-N, N)$ such that $w_{2}(\bar{\xi})=0$, then $w_{2}$ takes local minimum at $\bar{\xi}$ and the left hand side of the first inequality of (71) is zero at $\bar{\xi}$. We then have a contradiction.

Case 2. We may suppose that there is some point in $(-N, N)$ such that one of the components, say the $j$ th component, satisfies $\left(U_{1}^{\theta}(\xi)\right)_{j}<\left(U_{2}(\xi)\right)_{j}$ at that point, $j=1$ or 2 . We then increase $\theta$, that is, shift $U_{1}^{\theta}(\xi)$ further left, so that $U_{1}^{\theta}(-N)>$ $U_{2}(-N), U_{1}^{\theta}(N)>U_{2}(N)$. By the monotonicity of $U_{1}^{\theta}$ and $U_{2}$, we can find a $\bar{\theta} \in(0,2 N)$ such that in the interval $(-N, N)$ we have $U_{1}^{\theta}(\xi+\bar{\theta})>U_{2}(\xi)$. Shifting $U_{1}^{\theta}(\xi+\bar{\theta})$ back until one 
component of $U_{1}^{\theta}(\xi+\bar{\theta})$ first touches its counterpart of $U_{2}(\xi)$ at some point $\overline{\bar{\xi}} \in[-N, N]$, we then return back to Case 1 again, where it has been shown that this is impossible. Therefore, we must have

$$
U_{1}^{\theta}(\xi)>U_{2}(\xi)
$$

for all $\xi \in \mathbb{R}$, where $\theta$ is the one chosen by means of (66) as described above.

Now, decrease $\theta$ until one of the following situations happens.

(1) There exists a $\bar{\theta} \geq 0$, such that $U_{1}^{\bar{\theta}}(\xi) \equiv U_{2}(\xi)$. In this case, we have finished the proof.

(2) There exists a $\bar{\theta} \geq 0$ and $\xi_{1} \in \mathbb{R}$, such that one of the components of $U^{\bar{\theta}}$ and $U_{2}$ are equal there; for all $\xi \in$ $\mathbb{R}$, we have $U_{1}^{\bar{\theta}}(\xi) \geq U_{2}(\xi)$. On applying the maximum principle on $\mathbb{R}$ and using the same argument as we did for Case 1 , we see that this is impossible.

Consequently, in either situation, there exists a $\bar{\theta} \geq 0$, such that

$$
U_{1}^{\bar{\theta}}(\xi) \equiv U_{2}(\xi)
$$

for all $\xi \in \mathbb{R}$.

\section{References}

[1] A. J. Trewenack and K. A. Landman, "A traveling wave model for invasion by precursor and differentiated cells," Bulletin of Mathematical Biology, vol. 71, no. 2, pp. 291-317, 2009.

[2] D. Xu and X.-Q. Zhao, "Bistable waves in an epidemic model," Journal of Dynamics and Differential Equations, vol. 16, no. 3, pp. 679-707, 2004.

[3] X.-Q. Zhao and W. Wang, "Fisher waves in an epidemic model," Discrete and Continuous Dynamical Systems B, vol. 4, no. 4, pp. 1117-1128, 2004.

[4] P. K. Denman, D. L. S. McElwain, and J. Norbury, "Analysis of travelling waves associated with the modelling of aerosolised skin grafts," Bulletin of Mathematical Biology, vol. 69, no. 2, pp. 495-523, 2007.

[5] B. Kaźmierczak and V. Volpert, "Mechano-chemical calcium waves in systems with immobile buffers," Polish Academy of Sciences, vol. 60, no. 1, pp. 3-22, 2008.

[6] B. Kazmierczak and V. Volpert, "Travelling calcium waves in systems with non-diffusing buffers," Mathematical Models \& Methods in Applied Sciences, vol. 18, no. 6, pp. 883-912, 2008.

[7] B. Kaźmierczak and V. Volpert, "Calcium waves in systems with immobile buffers as a limit of waves for systems with nonzero diffusion,” Nonlinearity, vol. 21, no. 1, pp. 71-96, 2008.

[8] B. Kazmierczak and V. Volpert, "Travelling waves in partially degenerate reaction-diffusion systems," Mathematical Modelling of Natural Phenomena, vol. 2, no. 2, pp. 106-125, 2007.

[9] A. Ghazaryan, Y. Latushkin, S. Schecter, and A. J. de Souza, "Stability of gasless combustion fronts in one-dimensional solids," Archive for Rational Mechanics and Analysis, vol. 198, no. 3, pp. 981-1030, 2010.
[10] J. Fang and X.-Q. Zhao, "Monotone wavefronts for partially degenerate reaction-diffusion systems," Journal of Dynamics and Differential Equations, vol. 21, no. 4, pp. 663-680, 2009.

[11] H. Berestycki and L. Nirenberg, "Travelling fronts in cylinders," Annales de l'Institut Henri Poincaré, vol. 9, no. 5, pp. 497-572, 1992.

[12] A. W. Leung, X. Hou, and W. Feng, "Traveling wave solutions for Lotka-Volterra system re-visited," Discrete and Continuous Dynamical Systems B, vol. 15, no. 1, pp. 171-196, 2011.

[13] A. I. Volpert, V. A. Volpert, and V. A. Volpert, Traveling Wave Solutions of Parabolic Systems, vol. 140 of Translations of Mathematical Monographs, American Mathematical Society, Providence, RI, USA, 1994.

[14] D. H. Sattinger, "On the stability of waves of nonlinear parabolic systems," Advances in Mathematics, vol. 22, no. 3, pp. 312-355, 1976.

[15] W. A. Coppel, Dichotomies in Stability Theory, vol. 629 of Lecture Notes in Mathematics, Springer, Berlin, Germany, 1978. 


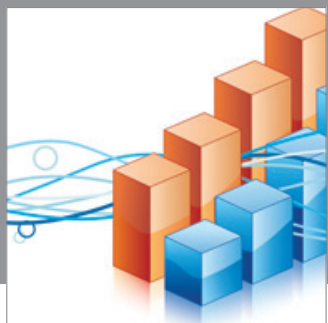

Advances in

Operations Research

mansans

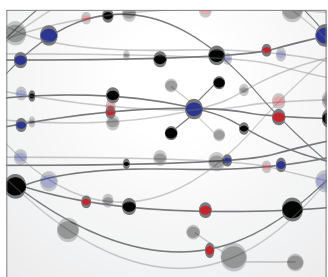

The Scientific World Journal
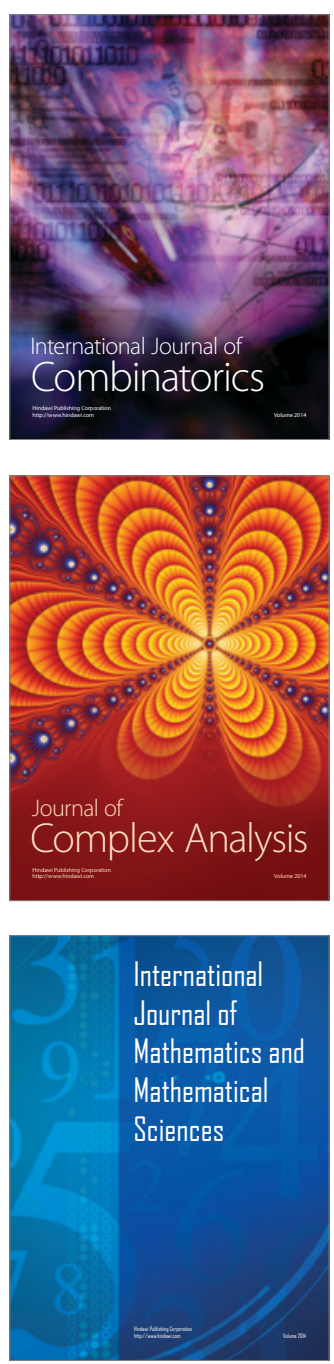
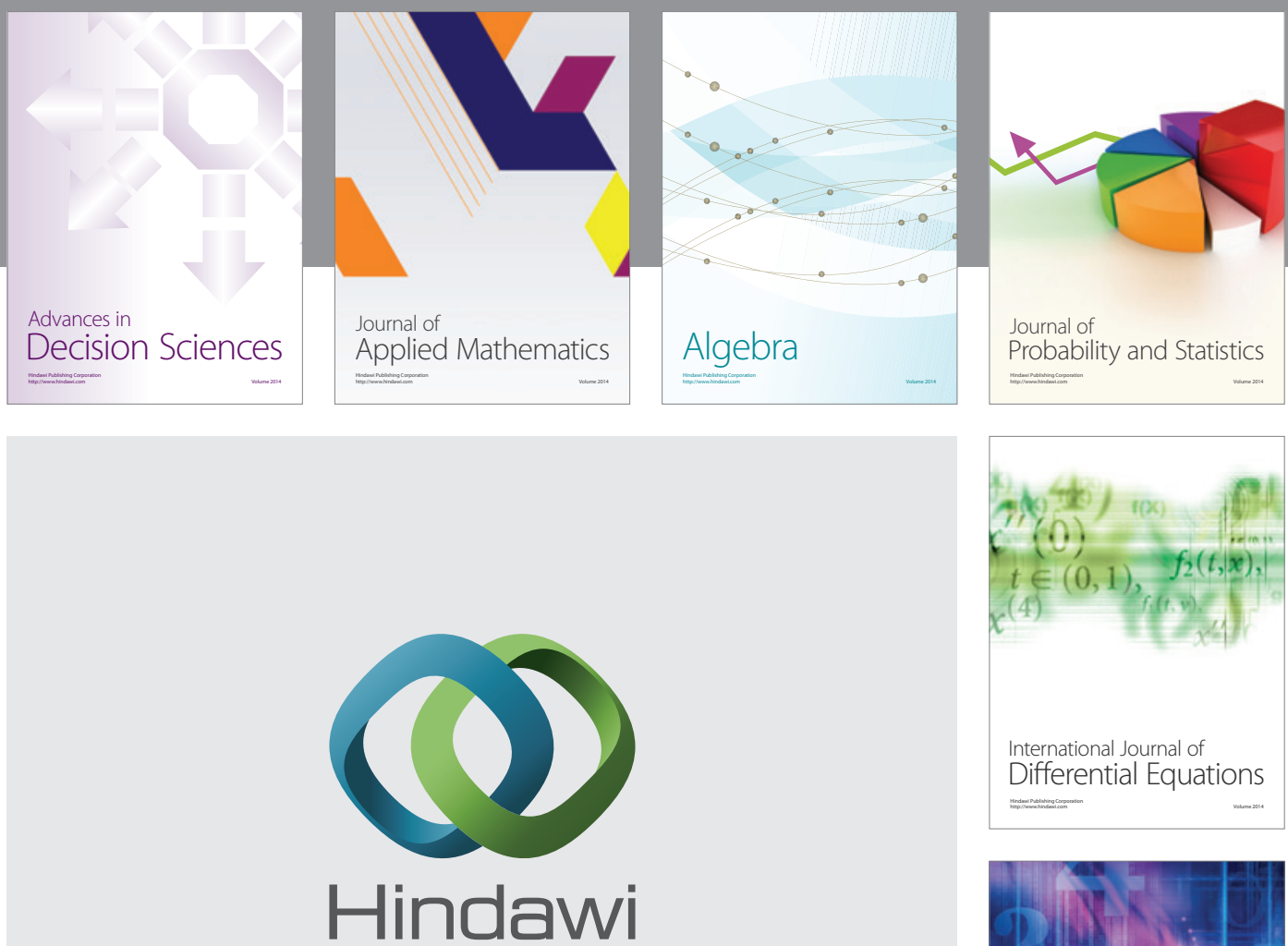

Submit your manuscripts at http://www.hindawi.com
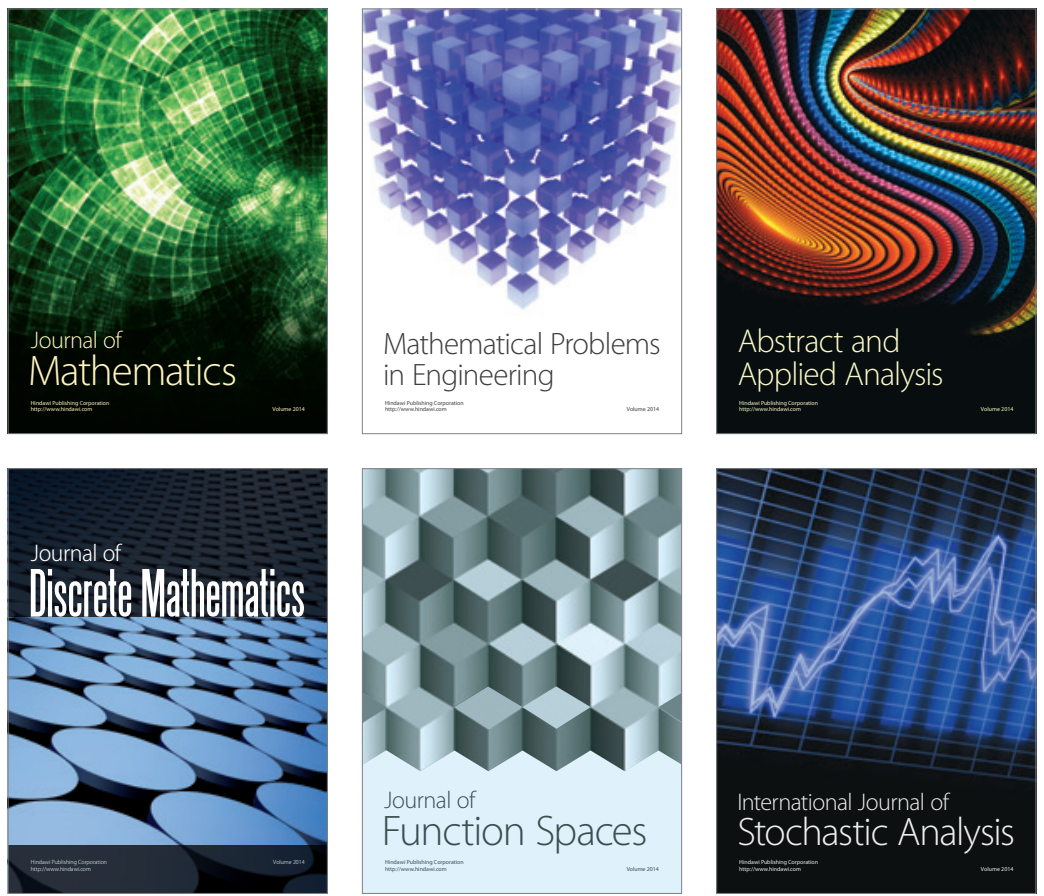

Journal of

Function Spaces

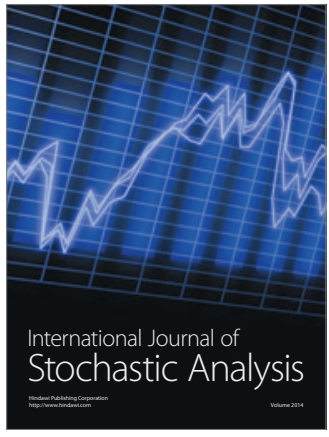

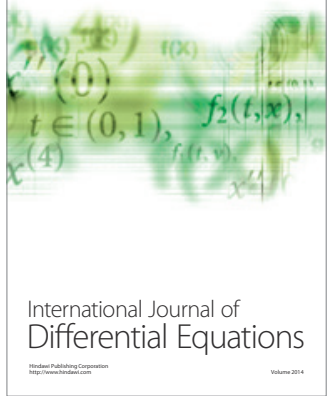
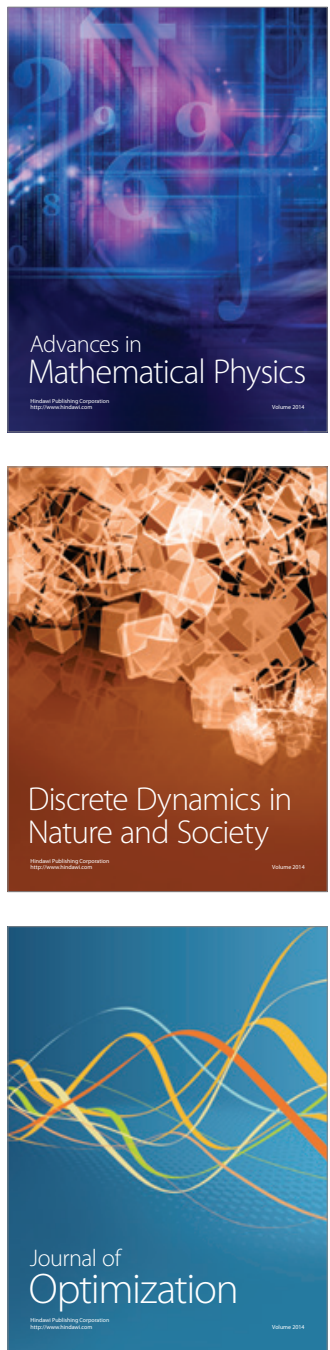